\title{
Учет подложки при расчете электрического сопротивления микродисковых лазеров
}

\author{
(C) А.Е. Жуков ${ }^{1}$, Н.В. Крыжановская ${ }^{1}$, Э.И. Моисеев ${ }^{1}$, А.М. Надточий ${ }^{1,3}$, М.В. Максимов ${ }^{2,1}$, А.С. Драгунова ${ }^{1}$ \\ ${ }^{1}$ Национальный исследовательский университет „Высшая школа экономики“, \\ 198008 Санкт-Петербург, Россия \\ ${ }^{2}$ Санкт-Петербургский национальный исследовательский Академический университет \\ им. Ж.И. Алфрёрова Российской академии наук, \\ 194021 Санкт-Петербург, Россия \\ ${ }^{3}$ Физико-технический институт им. А.Ф. Иоффе Российской академии наук, \\ 194021 Санкт-Петербург, Россия \\ E-mail: zhukale@gmail.com
}

Поступила в Редакцию 7 октября 2020 г.

В окончательной редакции 17 октября 2020 г.

Принята к публикации 17 октября 2020 г.

\begin{abstract}
Представлены аналитические выражения и с их помощью выполнен анализ компонент электрического сопротивления инжекционных микродисковых лазеров в зависимости от размера микродискового резонатора, параметров подложки и геометрии расположения контакта к ней.
\end{abstract}

Ключевые слова: микролазер, микродисковый резонатор, электрическое сопротивление, растекание тока, эффект скопления тока.

DOI: 10.21883/FTP.2021.02.50508.9530

\section{1. Введение}

В последнее время большой интерес привлекли микродисковые/микрокольцевые лазеры, которые рассматриваются как альтернатива вертикально-излучающим лазерам для систем оптической связи на короткие расстояния $[1,2]$. Достоинством подобных микролазеров является простота конструкции и возможность их интеграции, монолитной или гибридной, с подложками кремния [3,4]. Важным параметром микролазера является последовательное электрическое сопротивление. Его уменьшение ведет к увеличению коэффициента полезного действия, снижению тепловыделения и энергозатрат при переключении. Вклад сопротивления подложки, ввиду ее массивности, может оказаться существенным в общем последовательном сопротивлении микролазера. При формировании микролазера на основе слоев $p-i-n$-структуры, синтезированной на чужеродной подложке (например, на кремнии), интерфейс подложка-эпитаксиальные слои, как правило, обладает плохими электрическими свойствами, в связи с чем контакт формируется к сильно легированному буферному слою $\left(n^{+}\right.$-GaAs), осажденному поверх $\mathrm{Si}$ и предшествующему слоям лазерной гетероструктуры [5]. $\mathrm{B}$ случае микролазера на проводящей подложке $\mathrm{A}^{\mathrm{III}} \mathrm{B}^{\mathrm{V}}$ или его гибридной интеграции с кремнием, формирующая омический контакт с подложкой металлизация может быть нанесена как на сторону подложки, противоположную микрорезонатору (контакт снизу), так и на ту же, где расположен и микрорезонатор (контакт сверху).

В настоящей статье нами теоретически проанализировано влияние геометрии расположения контактов и параметров микролазера (радиус мезы, толщина и проводимость проводящего слоя подложки) на его электрическое сопротивление.

\section{2. Геометрия и параметры модели}

На рис. 1 схематически показаны варианты конструкции микродискового лазера с электрическим контактом снизу (рис. 1, $a$ ), занимающим всю обратную сторону подложки, либо сверху (рис. 1,b), покрывая поверхность вокруг основания микродиска на удалении $w$ от его края. При нижнем расположении контакта определенная толщина подложки $h_{\text {(sub_down) }}$ достигается за счет ее утончения, предшествующего напылению нижней контактной металлизации, и ограничена снизу пределом механической прочности ( 100 мкм). При верхнем расположении контакта следует различать два случая: когда подложка является полностью проводящей (в этом случае ее толщина $h_{\text {(sub_up) }} \geq 100$ мкм), либо когда контакт сформирован поверх проводящего слоя, синтезированного на непроводящей чужеродной подложке (в этом случае $h_{\text {(sub_up) }}$ может варьироваться от долей мкм до < 10 мкм). Для определенности, будем полагать подложку материалом $n$-типа, с одинаковой для всех типов геометрии контакта проводимостью $\sigma_{\mathrm{sub}}=400 \mathrm{OM}^{-1} \cdot \mathrm{cm}^{-1}$, что соответствует подавляющему большинству реальных случаев (например, подложки производителя „АXT Inc.“ $n^{+}-\mathrm{GaAs}(100)$ характеризуются концентрацией $(0.8-4) \cdot 10^{18} \mathrm{~cm}^{-3}$ и подвижностью $\left.(1-2.5) \cdot 10^{3} \mathrm{~cm}^{2} /(\mathrm{B} \cdot \mathrm{c})[6]\right)$.

Типичное значение $w$ составляет единицы мкм, в частности на микрофотографии, представленной на рис. 1, 

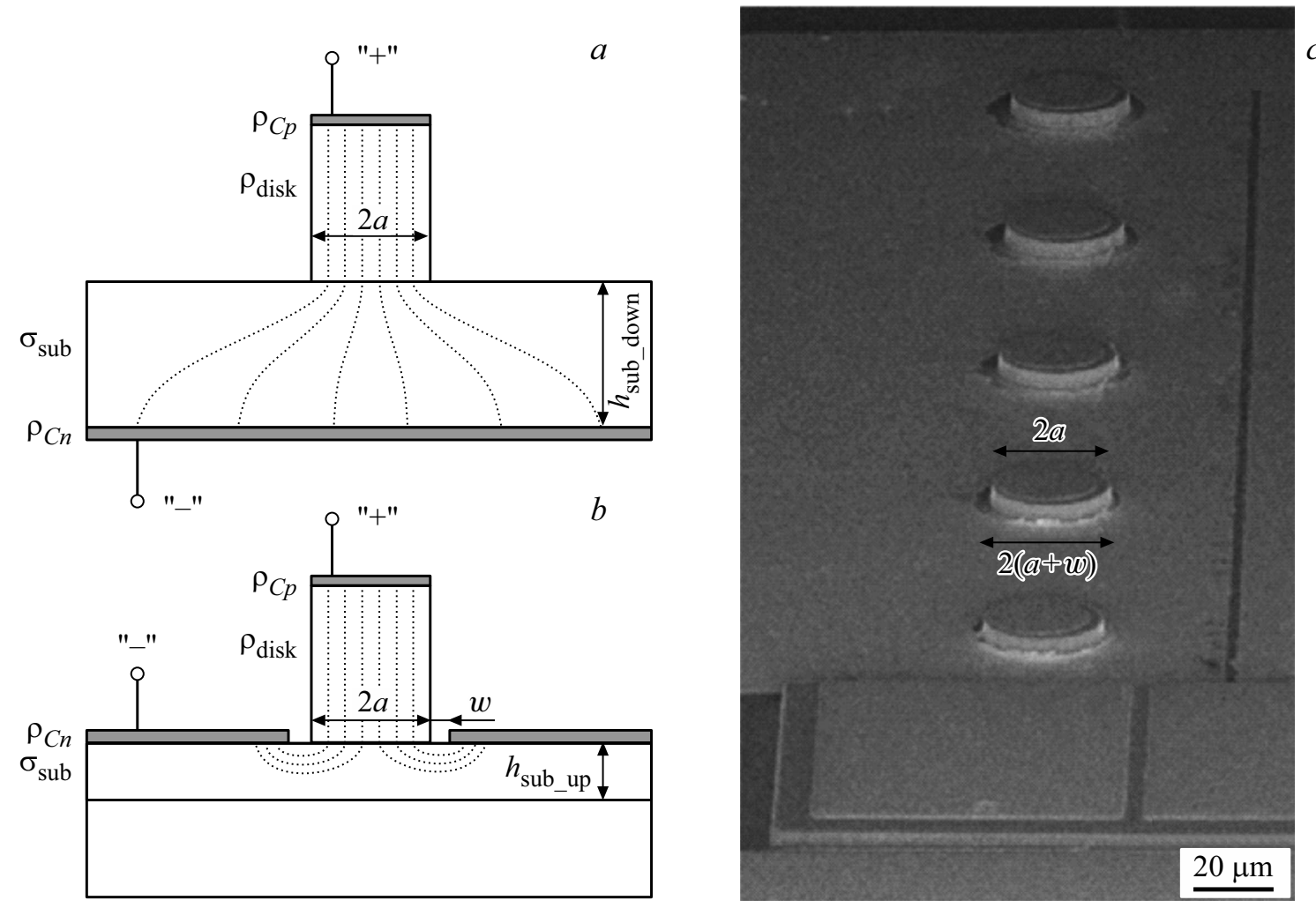

Рис. 1. Схематическое изображение микродискового лазера с нижним расположением контакта к проводящей подложке $(a)$ и с верхним расположением контакта к проводящему слою на непроводящей подложке $(b)$. Пунктиром показаны линии тока. Микрофотография микродисковых лазеров на кремниевой подложке с проводящим $n$-GaAs слоем с верхним расположением контакта к подложке $(c)$.

и изображающей несколько микродисковых лазеров диаметром $a=30$ мкм, изготовленных из гетероструктур с квантовыми точками, синтезированных на кремнии, величина $w \approx 2.5$ мкм. В дальнейшем в расчетах мы будем использовать эту величину зазора. Микродисковый резонатор имеет радиус $a$, который для общности анализа полагаем лежащим в широких пределах от единиц $\mu \mathrm{m}$, что отвечает наименьшим сообщенным размерам микродисковых лазеров, в которых наблюдалась лазерная генерация при комнатной температуре [7], до сотен мкм. Отметим, что типичные значения а в инжекционных микродисковых лазерах составляют мкм.

\section{3. Контакт снизу}

В этом случае полное сопротивление микролазера $R_{\text {down }}=R_{\text {disk }}+R_{\text {(sub_down) }}$ складывается из сопротивления $R_{\text {disk, }}$, вносимого непосредственно микродисковым резонатором и $R_{\text {(sub_down) }}$ - сопротивления подложки в этой геометрии. Величина $R_{\text {disk }}$ определяется последовательным сопротивлением всех слоев лазерной гетероструктуры (имеющих толщину $h_{i}$ и удельную проводимость $\sigma_{i}$ ), а также контактом $p$-типа, сформированным на вершине микродиска и характеризующимся удельным контактным сопротивлением $\rho_{C p}$. Их совокупный вклад может быть описан нормированным на площадь микродиска сопротивлением:

$$
\rho_{\text {disk }}=\left[\sum_{i} \frac{h_{i}}{\sigma_{i}}+\rho_{C p}\right], \quad R_{\text {disk }}=\frac{1}{\pi a^{2}} \rho_{\text {disk }} .
$$

Хотя, как будет показано далее, не все компоненты сопротивления масштабируются пропорционально площади мезы микролазера, удобно рассматривать не только абсолютные значения сопротивлений $R$, но и их нормированные на площадь значения $\rho \equiv \pi a^{2} R$. Это облегчает их сопоставление с величиной $\rho_{\text {disk }}$, определяемой лазерной гетероструктурой. В работе [8] сообщалось об образовании на боковой поверхности тонкого непроводящего слоя толщиной $\delta \sim 1$ мкм. В случае если $\delta \neq 0$ ниже, истинный радиус микродиска должен быть заменен на эффективный радиус проводимости $a-\delta$.

Типичные значения $\rho_{\text {disk }}$ близки к соответствующей величине в лазерах полосковой конструкции и для микродисковых лазеров InGaAs/GaAs/AlGaAs варьируются в диапазоне $(5-10) \cdot 10^{-5} \mathrm{OM}^{-c^{2}}[9,10]$. В дальнейшем будем считать $\rho_{\text {disk }}=8 \cdot 10^{-5} \mathrm{OM} \cdot \mathrm{cm}^{2}$. При этом омический контакт с подложкой характеризуется приблизительно на 2 порядка меньшим контактным сопротивлением [11]. Вследствие этого, а также большой площади нижнего контакта, определяемой размерами чипа 

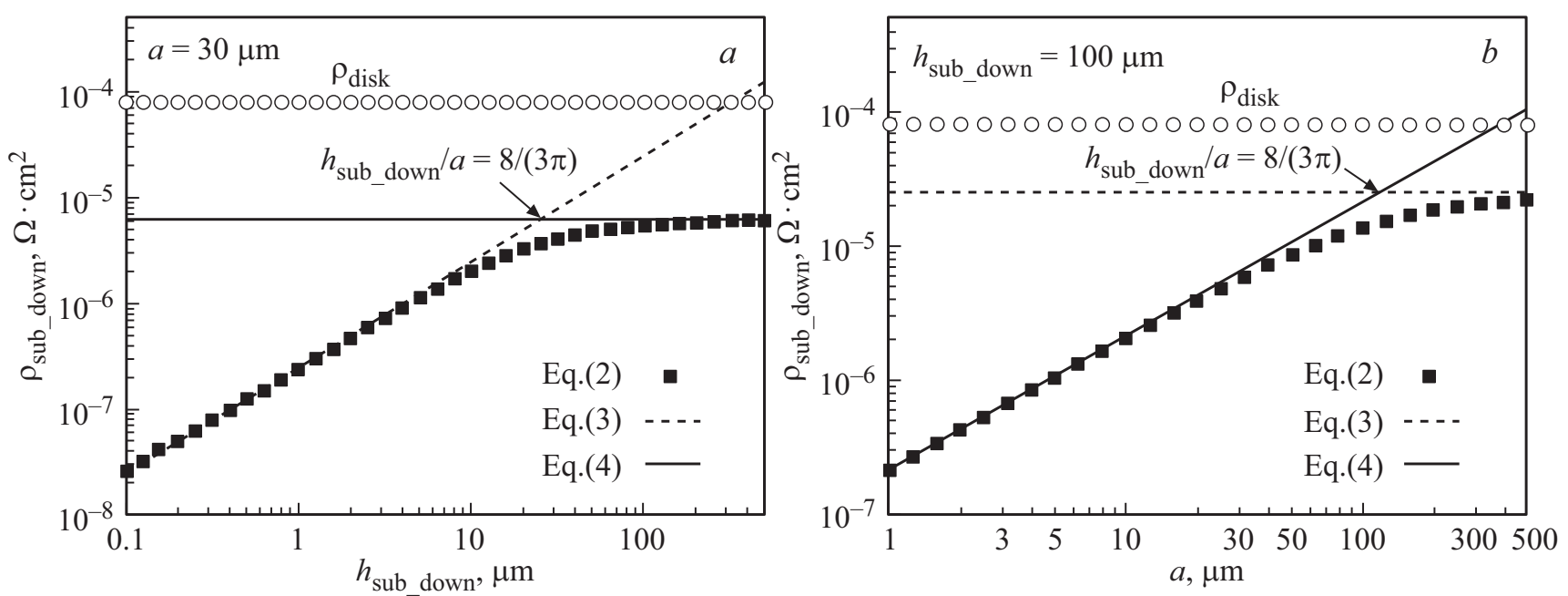

Рис. 2. Для расположения контакта снизу зависимость от толщины подложки $(a)$ или от радиуса диска $(b)$ нормированного сопротивления подложки (квадраты) и асимптоты для случаев $h_{\text {sub_down }} \ll a$ (штриховая линия) и $h_{\text {sub_down }} \gg a$ (сплошная линия). Круги - нормированное сопротивление мезы.

$(\mathrm{mm} \times \mathrm{mm}$ по порядку величины $)$, вкладом контактного сопротивления $n$-металла в сопротивление подложки в рассматриваемой геометрии можно пренебречь.

Сопротивление подложки соответствует геометрии задачи о сопротивлении растекания дискового контакта, рассмотренной в работе [12]:

$$
R_{\text {sub_down }}=\frac{1}{a \pi \sigma_{\text {sub }} / 2} \int_{0}^{\infty} \operatorname{th}\left(u \frac{h_{\text {sub_down }}}{a}\right)\left(\frac{J_{1}(u)}{u}\right)^{2} d u .
$$

Здесь $J_{1}-$ функция Бесселя 1-го рода 1-го порядка, $u$ - безразмерная переменная интегрирования. В предельных случаях:

$$
\begin{gathered}
\left(h_{\text {sub_down }} \ll a\right) \quad R_{\text {sub_down }}=\frac{1}{\pi a^{2}} \frac{h_{\text {sub_down }}}{\sigma_{\text {sub }}}, \\
\rho_{\text {sub_down }}=\frac{h_{\text {sub_down }}}{\sigma_{\text {sub }}} ; \\
\left(h_{\text {sub_down }} \gg a\right) \quad R_{\text {sub_down }}=\frac{8}{3 \pi^{2} a \sigma_{\text {sub }}} \cong \frac{1}{4 a \sigma_{\text {sub }}}, \\
\rho_{\text {sub_down }}=\frac{8 a}{3 \pi \sigma \mathrm{sub}} \cong \frac{a}{\sigma_{\text {sub }}} .
\end{gathered}
$$

Мы учли поведение гиперболического тангенса $(\operatorname{th}(z) \approx z$ или 1 при $z \rightarrow 0$ или $\infty), \quad$ а также что $\int_{0}^{\infty} \frac{1}{u}\left(J_{1}(u)\right)^{2} d u=1 / 2 \quad$ и $\quad \int_{0}^{\infty}\left(\frac{J_{1} u}{u}\right)^{2} d u=4 /(3 \pi)$. Выражение (3) по форме аналогично (1) и соответствует вертикальному пути тока (без бокового растекания), a (4) - сопротивлению растекания дискового контакта на полубесконечном проводящем слое [13].

На рис. 2, а показана зависимость $\rho_{\text {sub_down }}$ от толщины проводящей подложки, а на рис. $2, b$ от радиуса мезы. На обоих рисунках отчетливо видны два участка, соответствующих малым толщинам подложки / большим радиусам диска (выражение (3)) и большим толщинам подложки/малым радиусам диска (4). Граница двух режимов видна на рис. 2 достаточно отчетливо. Ее можно оценить, приравняв (3) и (4):

$$
h_{\text {sub_down }} / a=\frac{8}{3 \pi} \cong 1 \text {. }
$$

Как видно, она не зависит от проводимости материала и имеет место, когда толщина подложки приблизительно совпадает с радиусом микрорезонатора.

Поскольку на практике $h_{\text {sub_down }} \gtrsim 100$ мкм, для микрорезонаторов $(a \lesssim 20$ мкм $)$ реализуется приближение (4), т.е. нормированное на площадь сопротивление

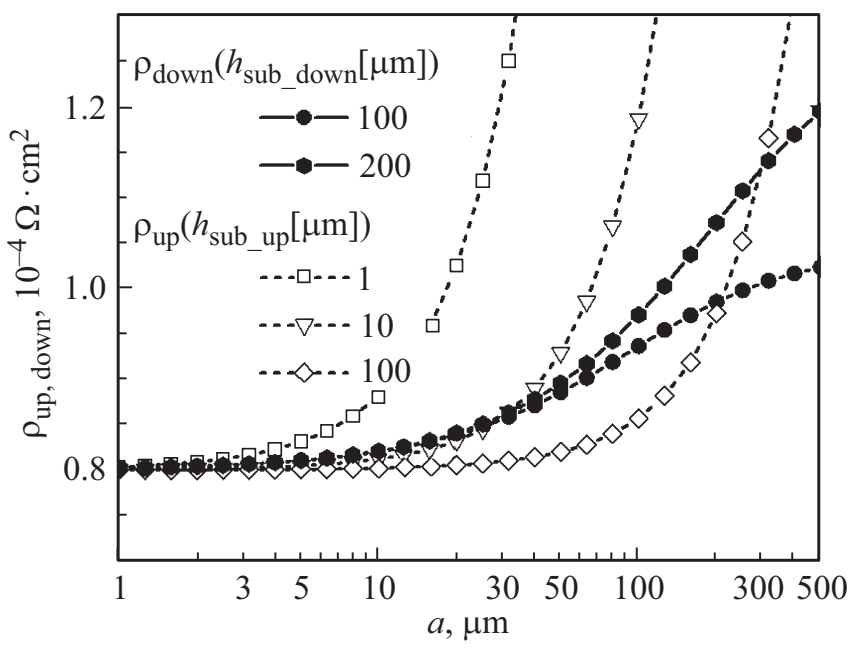

Рис. 3. Зависимость нормированного сопротивления микродискового лазера от его радиуса для расположения контакта снизу $\left(h_{\text {sub_down }}=100\right.$ мкм - круги, 200 мкм - шестиугольники) либо сверху $\left(h_{\text {sub_up }}=1\right.$ мкм - квадраты, 10 мкм треугольники, 100 мкм - ромбы). 
подложки практически не зависит от ее толщины и масштабируется пропорционально радиусу мезы. Поскольку нормированное сопротивление самой мезы остается неизменным, то $\rho_{\text {sub_down }} / \rho_{\text {disk }} \propto a$, т. е. подложка вносит все меньший вклад в полное сопротивление микролазера при уменьшении его диаметра. В отличие от этого, в макролазерах $(a \gtrsim 50$ мкм) влияние подложки существенно, но оно может быть снижено за счет утончения подложки (рис. 3). В целом полное сопротивление $\rho_{\text {down }}$ достаточно слабо зависит от радиуса, изменяясь от $\rho_{\text {disk }}$ в области малых радиусов до $\rho_{\text {disk }}+h_{\text {sub_down }} / \sigma_{\text {sub }}$ для больших радиусов.

\section{4. Контакт сверху, сравнение}

В случае размещения контакта к подложке на одной стороне с микродиском уменьшение толщины проводящей подложки ведет не к снижению, а к увеличению ее сопротивления, поскольку реализуется преимущественно радиальный путь тока через проводящую часть подложки (рис. $1, b)$. При этом ток через контакты эффективно протекает лишь вблизи их краев, наиболее близких друг другу. Это явление получило название эффекта скопления тока (current crowding effect); он оказывает, в том числе, влияние на характеристики вертикально-излучающих лазеров [14].

Полное сопротивление для расположения контакта сверху $R_{\text {up }}=R_{\text {sep }}+R_{\text {in }}+R_{\text {out }}$ складывается из сопротивлений полупроводникового промежутка $R_{\mathrm{sep}}$, внутреннего контакта $R_{\text {in }}$ и внешнего (охватывающего) контакта $R_{\text {out }}[15]$ :

$$
R_{\text {sep }}=\frac{r}{2 \pi} \ln \left(\frac{a+w}{a}\right), \quad R_{\text {in,out }}=\frac{r}{2 \pi \frac{a_{C}}{L_{T}}} \frac{Z_{0}\left(\frac{a_{C}}{L_{T}}\right)}{Z_{1}\left(\frac{a_{C}}{L_{T}}\right)} .
$$

Здесь $Z_{0,1} \equiv I_{0,1}$ либо $K_{0,1}$ - модифицированные функции Бесселя 1-го рода (для внутреннего контакта) или 2-го рода (для внешнего контакта) 0-го и 1-го порядков, $a_{C}-$ радиус контакта ( $a$ либо $a+w$ соответственно), $L_{T}=\sqrt{\rho_{C} / r}-$ так называемая длина передачи (transfer length), характеризующая эффективный размер области контакта, через которую ток входит в полупроводниковый слой при рассматриваемой геометрии и определяемая как листовым $(\mathrm{OM} / \square)$ сопротивлением $r$ полупроводника, через который протекает ток, так и удельным контактным сопротивлением $\rho_{C}$. Если бы последнее было одинаковым как для внутренней, так и для внешних контактных областей, мы получили бы известное выражение [16] для полного сопротивления контакта металл-полупроводниковый слой - металл циркулярной тестовой структуры, используемой для измерения контактного сопротивления (CTLM) [17]:

$$
R=\frac{r}{2 \pi}\left[\ln \left(1+\frac{w}{a}\right)+\frac{L_{T}}{a} \frac{I_{0}\left(\frac{a}{L_{T}}\right)}{I_{1}\left(\frac{a}{L_{T}}\right)}+\frac{L_{T}}{a+w} \frac{K_{0}\left(\frac{a+w}{L_{T}}\right)}{K_{1}\left(\frac{a+w}{L_{T}}\right)}\right] .
$$

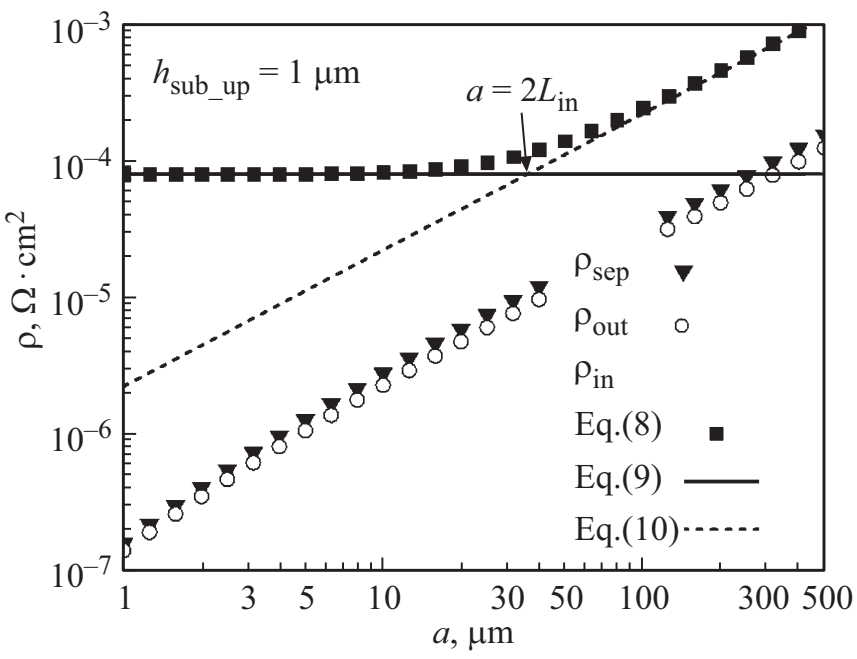

Рис. 4. Для контакта сверху и $h_{\text {sub_up }}=1$ мкм зависимость от радиуса слагаемых нормированного сопротивления: межконтактного промежутка (треугольники), внешнего контакта (круги) и внутреннего контакта (квадраты), а также асимптоты для малых радиусов (сплошная линия) и больших радиусов (пунктирная линия).

Однако для рассматриваемого нами случая микродискового лазера нельзя считать обе контактные площадки, внутреннюю и внешнюю, одинаковыми с точки зрения длины переноса, поскольку для внутренней области роль $\rho_{C}$ играет нормированное на площадь последовательное сопротивление микродискового резонатора $\rho_{\text {disk. Таким }}$ образом, для нормированных на площадь мезы компонент сопротивления $\rho_{\text {up }} \equiv \pi a^{2} R_{\text {up }}=\rho_{\text {sep }}+\rho_{\text {in }}+\rho_{\text {out }}$, могут быть записаны следующие выражения

$$
\begin{gathered}
\rho_{\text {sep }}=\frac{r_{\text {sub }}}{2} a^{2} \ln \left(1+\frac{w}{a}\right), \quad \rho_{\text {in }}=\frac{r_{\text {sub }}}{2} a L_{\text {in }} \frac{I_{0}\left(\frac{a}{L_{\text {in }}}\right)}{I_{1}\left(\frac{a}{L_{\text {in }}}\right)}, \\
\rho_{\text {out }}=\frac{r_{\text {sub }}}{2} \frac{a^{2} L_{\text {in }}}{a+w} \frac{K_{0}\left(\frac{a+w}{L_{\text {out }}}\right)}{K_{1}\left(\frac{a+w}{L_{\text {out }}}\right)},
\end{gathered}
$$

где $r_{\text {sub }}=1 /\left(h_{\text {sub_up }} \sigma_{\text {sub }}\right)-$ листовое сопротивление проводящего слоя подложки, $L_{\text {out }}=\sqrt{\rho_{C n} / r_{\text {sub }}}$ и $L_{\text {in }}=\sqrt{\rho_{\text {disk }} / r_{\text {sub }}}$. Для $h_{\text {sub_up }}=1$ мкм имеем $L_{\text {out }}$ и $L_{\text {in }}=2$ и 18 мкм соответственно. Зависимость нормированных на площадь мезы компонент сопротивления от радиуса приведена на рис. 4. Как видно, при использованном в расчетах (типичном для микродисковых лазеров) наборе параметров заметно преобладает слагаемое $\rho_{\text {in }}$.

Для мез большого радиуса $\left(a \gg L_{\text {in }}\right)$ сопротивление микродискового лазера аналогично сопротивлению проводника шириной, отвечающей периметру мезы, и длиной, равной сумме обеих длин переноса и промежутка между контактами:

$$
\begin{gathered}
R_{\text {up }} \approx \frac{r_{\text {sub }}}{2 \pi a}\left(w+L_{\text {in }}+L_{\text {out }}\right) \approx \frac{r_{\text {sub }}}{2 \pi a} L_{\text {in }}, \\
\rho_{\text {up }} \approx \rho_{\text {in }}=\frac{r_{\text {sub }}}{2} a L_{\text {in }} .
\end{gathered}
$$


Мы учли, что $Z_{0}(z) / Z_{1}(z) \approx 1$ при $z \rightarrow \infty$, а также то, что $L_{\text {in }}$ превосходит как $L_{\text {out }}$, так и $w$. Для малых радиусов микродиска $\left(a \ll L_{\text {in }}\right)$ асимптотическое поведение можно отыскать, учитывая, что $z \frac{I_{0}(z)}{I_{1}(z)} \approx 2$ при $z \rightarrow 0$.

$$
\rho_{\text {in }}=r_{\text {sub }} L_{\text {in }}^{2}=\rho_{\text {disk }}
$$

что соответствует нормированному сопротивлению собственно микродиска при нижнем расположении контакта к подложке (выражение (1)).

Сравнивая сопротивление для различных случаев расположения контакта к подложке (рис. 3), мы, таким образом, можем заключить, что при малых размерах мезы микродискового лазера $\left(a \ll L_{\text {in }}, a \ll h_{\text {sub_down }}\right.$ его сопротивление независимо от расположения контактов приблизительно одинаково и определяется сопротивлением слоев, образующих микродисковый резонатор $\left(\rho_{\text {up }} \approx \rho_{\text {down }} \approx \rho_{\text {disk }}\right)$. При верхнем расположении контакта резкий рост сопротивления начинается, когда радиус диска начинает превышать длину переноса $L_{\text {in }}$, зависящую от толщины проводящего слоя. Если $h_{\text {sub_down }} \sim 1$ мкм, это означает, что уже начиная с радиусов $\sim 20$ мкм отношение сопротивлений $\rho_{\text {up }} / \rho_{\text {down }} \approx r_{\text {sub }} a L_{\text {in }} /\left(2 \rho_{\text {disk }}\right)=a /\left(2 L_{\text {in }}\right)$ оказывается заметно больше единицы. Однако, если $h_{\text {sub_down }} \sim 100$ мкм (проводящая подложка), величина $L_{\text {in }}$ возрастает до $\sim 200$ мкм. В этом случае для радиусов микродиска, меньших этого размера, $\rho_{\text {up }}$ все еще приблизительно равно $\rho_{\text {disk }}$, тогда как при нижнем расположении контакта сопротивление подложки уже дает заметный вклад в $\rho_{\text {down }}$, так что $\rho_{\text {up }} / \rho_{\text {down }}$ оказывается меньше 1.

\section{5. Заключение}

Таким образом, нами аналитически исследовано электрическое сопротивление микродисковых лазеров для двух характерных случаев размещения контакта $n$-типа: снизу проводящей подложки, либо вокруг мезы микродиска на верхней стороне подложки (проводящей или с тонким проводящим слоем сверху). Физическими процессами, влияющими на величину сопротивления в той или иной геометрии, являются растекание тока в подложке и эффект скопления тока вблизи краев контактов. Для дисков большого размера с контактом сверху вклад подложки в полное сопротивление растет пропорционально радиусу и потому является определяющим. При расположении контакта снизу вклад подложки насыщается с ростом радиуса, но является существенным по сравнению с сопротивлением собственно диска. Для дисков предельно малого размера сопротивление фактически не зависит от расположения контакта к подложке, поскольку определяется сопротивлением слоев мезы. Для промежуточных размеров мезы, наиболее интересных с практической точки зрения (10-40мкм), сопротивление при расположении контакта сверху тонкого слоя поверх непроводящей подложки оказывается заметно больше, чем при его расположении снизу проводящей подложки. Однако если при верхнем расположении контакта используется проводящая подложка, сопротивление для микродисков таких размеров оказывается меньше, чем для традиционного размещения контакта снизу подложки. Это открывает путь для оптимизации электрических характеристик микродисковых лазеров, в том числе гибридно-интегрированных с кремнием.

\section{Финансирование работы}

Работа выполнена при поддержке проекта РНФ 19-7230010. Структурные исследования микродисков выполнены в рамках Программы фундаментальных исследований НИУ ВШЭ.

\section{Конфликт интересов}

Авторы заявляют, что у них нет конфликта интересов.

\section{Список литературы}

[1] Y. Wan, D. Inoue, D. Jung, J.C. Norman, C. Shang, A.C. Gossard, J.E. Bowers. Photon. Res., 6 (8), 776 (2018).

[2] F. Zubov, M. Maximov, N. Kryzhanovskaya, E. Moiseev, M. Muretova, A. Mozharov, N. Kaluzhnyy, S. Mintairov, M. Kulagina, N. Ledentsov, jr., L. Chorchos, N. Ledentstsov, A. Zhukov. Optics Lett., 44 (22), 5442 (2019).

[3] Y. Wan, J. Norman, Q. Li, M.J. Kennedy, D. Liang, C. Zhang, D. Huang, Z. Zhang, A.Y. Liu, A. Torres, D. Jung, A.C. Gossard, E.L. Hu, K.M. Lau, J.E. Bowers. Optica, 4 (8), 940 (2017).

[4] A.E. Zhukov, N.V. Kryzhanovskaya, E.I. Moiseev, A.S. Dragunova, M. Tang, S. Chen, H. Liu, M.M. Kulagina, S.A. Kadinskaya, F.I. Zubov, A.M. Mozharov, M.V. Maximov. Materials, 13 (10), 2315 (2020).

[5] N. Kryzhanovskaya, E. Moiseev, Yu. Polubavkina, M. Maximov, M. Kulagina, S. Troshkov, Yu. Zadiranov, Yu. Guseva, A. Lipovskii, M. Tang, M. Liao, J. Wu, S. Chen, H. Liu, A. Zhukov. Optics Lett., 42 (17), 3319 (2017).

[6] Semi-conducting GaAs Specifications, http://www.axt.com/site/index.php?q=node/37

[7] M.V. Maximov, N.V. Kryzhanovskaya, A.M. Nadtochiy, E.I. Moiseev, I.I. Shostak, A.A. Bogdanov, Z.F. Sadrieva, A.E. Zhukov, A.A. Lipovskii, D.V. Karpov, J. Laukkanen, J. Tommila. Nanoscale Res. Lett., 9 (1), 657 (2014).

[8] Ф.И. Зубов, Э.И. Моисеев, Г.О. Корнышов, Н.В. Крыжановская, Ю.М. Шерняков, А.С. Паюсов, М.М. Кулагина, Н.А. Калюжный, С.А. Минтаиров, М.В. Максимов, A.Е. Жуков. Письма ЖТФ, 45 (19), 37 (2019).

[9] A.S. Novikov, E.I. Moiseev, N.V. Kryzhanovskaya, B.I. Afinogenov, Y.A. Guseva, K. Kotlyar, A.A. Lipovskii, A.G. Nasibulin, M.V. Maximov, A.E. Zhukov. J. Phys. Conf. Ser., 1124, 041012 (2018).

[10] A.E. Zhukov, N. V. Kryzhanovskaya, E.I. Moiseev, A.M. Nadtochiy, A.S. Dragunova, M.V. Maximov, F.I. Zubov, S.A. Kadinskaya, Yu. Berdnikov, M.M. Kulagina, S.A. Mintairov, N.A. Kalyuzhnyy. IEEE J. Quant. Electron., 56 (5), 2000908 (2020).

[11] Т.В. Бланк, Ю.А. Гольдберг. ФТП, 41 (11), 1281 (2007). 
[12] Н.Н. Поляков, В.Л. Коньков. Изв. вузов. Физика, 9, 100 (1970).

[13] R.S. Timsit. In: Electrical Contacts: Principles and Applications, ed. by P.G. Sladev. 2nd edn (N.Y., USA, CRC Press, 2014) chap. 1, p. 3.

[14] V. Lysak, K.S. Chang, Y.-T. Lee. Appl. Phys. Lett., 87, 231118 (2005).

[15] S.S. Cohen, G. Gildenblat. In: Metal-Semiconductor Contacts and Devices, v. 13 (London, UK, 1986, Academic Press, 1986) chap. 4, p. 87.

[16] В.Я. Нисков, Г.А. Кубецкий. ФТП, 4 (9), 1806 (1970).

[17] G.S. Marlow, M.B. Das. Solid-State Electron., 25 (2), 91 (1982).

Редактор Г.А. Оганесян

\section{Impact of substrate in calculating the electrical resistance of microdisk lasers}

A.E. Zhukov' ${ }^{1}$, N.V. Kryzhanovskaya ${ }^{1}$, E.I. Moiseev' ${ }^{1}$, A.M. Nadtochiy ${ }^{1,3}$, M.V. Maximov ${ }^{2,1}$, A.S. Dragunova ${ }^{1}$

${ }^{1}$ National Research University Higher School

of Economics,

190008 St. Petersburg, Russia

${ }^{2}$ Alferov St. Petersburg Academic University,

194021 St. Petersburg, Russia

3 loffe Institute,

194021 St. Petersburg, Russia

Abstract Analytical expressions are presented and used to analyze the components of the electrical resistance of injection microdisk lasers depending on the size of the microdisk resonator, the parameters of the substrate, and the geometry of the contact to it. 